\title{
Adaptive Hybrid Function Projective Synchronization of Chaotic Systems with Time-Varying Parameters
}

\author{
Jinsheng Xing \\ School of Mathematics and Computer Science, Shanxi Normal University, Shanxi, Linfen 041004, China \\ Correspondence should be addressed to Jinsheng Xing, xjs6448@126.com
}

Received 11 November 2011; Accepted 7 February 2012

Academic Editor: Teh-Lu Liao

Copyright $@ 2012$ Jinsheng Xing. This is an open access article distributed under the Creative Commons Attribution License, which permits unrestricted use, distribution, and reproduction in any medium, provided the original work is properly cited.

The adaptive hybrid function projective synchronization (AHFPS) of different chaotic systems with unknown time-varying parameters is investigated. Based on the Lyapunov stability theory and adaptive bounding technique, the robust adaptive control law and the parameters update law are derived to make the states of two different chaotic systems asymptotically synchronized. In the control strategy, the parameters need not be known throughly if the time-varying parameters are bounded by the product of a known function of $t$ and an unknown constant. In order to avoid the switching in the control signal, a modified robust adaptive synchronization approach with the leakage-like adaptation law is also proposed to guarantee the ultimately uni-formly boundedness (UUB) of synchronization errors. The schemes are successfully applied to the hybrid function projective synchronization between the Chen system and the Lorenz system and between hyperchaotic Chen system and generalized Lorenz system. Moreover, numerical simulation results are presented to verify the effectiveness of the proposed scheme.

\section{Introduction}

Since the idea of synchronizing two identical autonomous chaotic systems under different initial conditions was first introduced in 1990 by Pecora and Carroll [1], chaos synchronization has been widely studied in physics, secure communication, chemical reactor, biological networks, and artificial neural networks. Up to now, different types of synchronization phenomena have been presented such as complete synchronization (CS) [2], generalized synchronization(GS) [3], lag synchronization [4], anticipated synchronization [5], phase synchronization [6], and antiphase synchronization [7], just to name a few. Also many control schemes such as the OGY method [8], delayed feedback method [9], 
adaptive control method [10], and impulsive control approach [11] have been employed to synchronize chaotic systems with different initial conditions.

Among all kinds of chaos synchronization schemes, projective synchronization, characterized by a scaling factor that two systems synchronize proportionally, has been extensively investigated by many authors $[12,13]$. This is because it can obtain faster communication with its proportional feature. However, most of investigations have concentrated on the case of constant scaling factor. Recently, a new kind of synchronization function projective synchronization (FPS) was introduced by Du et al. [14]. Function projective synchronization is the more general definition of projective synchronization. As compared with projective synchronization, function projective synchronization means that the drive and response systems could be synchronized up to a scaling function, which is not a constant. This characteristic could be used to get more secure communication in application to secure communications. This is because the unpredictability of the scaling function in FPS can enhance the security of communication.

On the other hand, chaotic systems are unavoidably exposed to an environment which may cause their parameters to vary within certain ranges such as environment temperature, voltage fluctuation, and mutual interfere among components. The system parameters may drift around their nominal values. As a result, in the studies [15-19] on control and synchronization of chaos, the problem of parametric uncertainty is a very significant and challenging one. In these researches, the most common method used to solve the parametric uncertainties is adaptive control schemes in which the unknown system parameters are updated adaptively according to certain rules. For example, in [15-18], it was assumed that the parameters of the driving system were totally uncertain or unknown to the response system. And the parameters of the response system can be different from those of the driving system. Some studies suppose that the parameters of the driving and the response systems are identical, but there are also some parametric uncertainties or perturbations. In [19], a nonlinear control method based on Lyapunov stability theorem was proposed to design an adaptive controller for synchronizing two different chaotic systems. It was assumed that the unknown parameters of the drive and the response chaotic systems were time varying. It is shown that the proposed scheme can identify the system parameters if the system parameters are time invariant and the richness conditions are satisfied. In [20], a special full-state hybrid projective synchronization type was proposed. The antisynchronization and complete synchronization could be achieved simultaneously in this new synchronization phenomenon. In [21], a hybrid projective synchronization (HPS), in which the different state variables can synchronize up to different scaling factors, was numerically observed in coupled partially linear chaotic complex nonlinear systems without adding any control term. In [22], the full-state hybrid projective synchronization (FSHPS) of chaotic and hyperchaotic systems was investigated with fully unknown parameters. Based on the Lyapunov stability theory, a unified adaptive controller and parameters update law were designed for achieving the FSHPS of chaotic and/or hyperchaotic systems with the same and different order. For two chaotic systems with different order especially, reduced-order MFSHPS (an acronym for modified full-state hybrid projective synchronization) and increased-order MFSHPS were studied. In [20-22], hybrid projective synchronization approaches assumed that the scaling factors were constants. In [23], a modified function projective synchronization between hyperchaotic Lorenz system and hyperchaotic Lu system was investigated by using adaptive method. By Lyapunov stability theory, the adaptive control law and the parameter update law were derived to make the state of two hyperchaotic systems modified function projective 
synchronized. However, in [23], the parameters update law was related to the unknown parameters, which will lead to infeasibility in engineering applications [24].

Motivated by the aforementioned discussion, we will formulate the hybrid function projective synchronization (HFPS) problem of different chaotic systems with unknown timevarying parameters. A robust adaptive synchronization method is proposed. By adding a compensator in the input vector to deal with time-varying parameters uncertainties by adaptive bounding technique, the uncertainties of the parameters in the Lyapunov function are eliminated. And by a parameter updating law, the nominal value of the unknown time-varying parameters and upper bound of uncertainty can be estimated. Based on the Lyapunov stability theory, this controller can achieve the robust adaptive synchronization of a class of chaotic system with time-varying unknown parameters. Some typical chaotic and hyper-chaotic systems are taken as examples to illustrate our technique.

The rest of this paper is organized as follows. In Section 2, the definition of HFPS is introduced. In Section 3, the general method of AHFPS is studied. In Section 4, two numerical examples are used to confirm the effectiveness of the proposed scheme. The conclusions are discussed in Section 5.

Notations. Throughout this paper, the notation $P^{\mathrm{T}}$ denotes the transpose of a vector $P$, while for $x \in R^{n}$, the notation $\|x\|=\sqrt{\left(x^{\mathrm{T}} x\right)}$ stands for the Euclidean norm of the vector $x$.

\section{The Definition of HFPS}

The drive system and the response system are defined as follows:

$$
\begin{gathered}
\dot{x}=f(x, t), \\
\dot{y}=g(y, t)+u(x, y, t),
\end{gathered}
$$

where $x, y \in R^{n}$ are the state vectors, $f, g: R^{n} \rightarrow R^{n}$ are continuous nonlinear vector functions, and $u(x, y, t)$ is the control vector.

We describe the error term

$$
e(t)=x-H(t) y,
$$

where $H(t)=\operatorname{diag}\left\{h_{1}(t), h_{2}(t), \ldots, h_{n}(t)\right\}$ is a scaling function matrix, $h_{i}(t)$ is a continuously differentiable bounded function, and $h_{i}(t) \neq 0$ for all $t$.

Definition 2.1 (HFPS). For two different systems described by (2.1), we say they are globally hybrid function projective synchronous (HFPS) with respect to the scaling function matrix $H(t)$ if there exists a vector controller $u(x, y, t)$ such that all trajectories $(x(t), y(t))$ in (2.1) with any initial conditions $(x(0), y(0))$ in $R^{n} \times R^{n}$ approach the manifold $E=\{(x(t), y(t))$ : $x(t)=H(t) y(t)\}$ as time $t$ goes to infinity, that is to say, $\lim _{t \rightarrow \infty}\|e(t)\|=\lim _{t \rightarrow \infty} \| x(t)-$ $H(t) y(t) \|=0$. This implies that the error dynamical system between the drive system and response system is globally asymptotically stable.

Remark 2.2. From the definition of HFPS, we can find that the definition of hybrid function projective synchronization includes function projective synchronization when the 
scaling function matrix $H(t)=\alpha(t) I$ with $\alpha(t)$ being function of $t$ and general projective synchronization when $H(t)=\operatorname{diag}\left\{h_{1}(t), h_{2}(t), \ldots, h_{n}(t)\right\}$ with $h_{i}$ being constant.

\section{Design of the General Scheme of AHFPS}

Consider a class of chaotic system with unknown time-varying parameters, which is described by

$$
\dot{x}=A x+f(x)+D(x) \theta(t),
$$

where $x \in R^{n}$ is the state vector $A \in R^{n \times n}$ and $f(x): R^{n} \rightarrow R^{n}$ are the linear coefficient matrix and nonlinear part of system (3.1), respectively. $D(x): R^{n} \rightarrow R^{n \times p}$, and $\theta(t)=(\Phi+\Delta \Phi(t)) \in$ $R^{p}$ is the uncertain parameter vector. Here $\Phi$ is the nominal value of $\theta(t)$, and $\Delta \Phi(t)$ is the uncertainty or disturbance. Equation (3.1) is considered as the drive system. The response system with a controller $u(x, y, t) \in R^{n}$ is introduced as follows:

$$
\dot{y}=B y+g(y)+u(x, y, t),
$$

where $y \in R^{n}$ is the state vector, $B \in R^{n \times n}$ and $g(y): R^{n} \rightarrow R^{n}$ are the linear coefficient matrix and a continuous nonlinear vector function, and $u(x, y, t)$ is the control vector.

Definition 3.1 (AHFPS). For two different systems described by (3.1) and (3.2), we say they are globally adaptive hybrid function projective synchronous (AHFPS) with respect to the scaling function matrix $H(t)$ if there exists a vector adaptive controller $u(x, y, \widehat{\theta}, t)$ and parameters update law such that all trajectories $(x(t), y(t)), \hat{\theta}(t)$ in (3.1) and (3.2) with any initial conditions $(x(0), y(0), \widehat{\theta}(0))$ in $R^{n} \times R^{n} \times R^{p}$ approach the manifold $E=\{(x(t), y(t))$ : $x(t)=H(t) y(t)\}$ as time $t$ goes to infinity, and $\widehat{\theta}(t)$ is bounded, that is to say, $\lim _{t \rightarrow \infty}\|e(t)\|=$ $\lim _{t \rightarrow \infty}\|x(t)-H(t) y(t)\|=0$.

Remark 3.2. The system (3.1) studied in this paper depends linearly on the unknown timevarying parameters, although $D(x)$ is a known nonlinear function matrix of state vector. The class of nonlinear dynamical systems includes an extensive variety of chaotic systems such as Lorenz system, the Rössler system, the Duffing system, Chua's circuit, the generalized Lorenz system.

Remark 3.3. Here, in general, (3.1) and (3.2) are different chaotic systems, we will investigate the AHFPS of nonidentical chaotic systems. When $B=A$ and $g=f+D(x) \theta(t)$, the synchronization mentioned above is the AHFPS of identical chaotic systems.

Assumption 3.4. The norm of $\Delta \Phi(t)$ satisfies the following inequality:

$$
\|\Delta \Phi(t)\| \leq M \vartheta(t)
$$

for all $t \in R^{+}$, where $M \in R^{+}$is the unknown constant parameter and $\vartheta(t)$ is a known continuous function of $t$. 
Remark 3.5. The condition in Assumption 3.4 only requires that the norm of time-varying parameters has an upper bound, which is the product of a known function of $t$ and an unknown constant, this condition is relaxed as the norm of time-varying parameters has an unknown constant upper bound stated in many papers, such as [19], just to name a few.

The dynamic equation of synchronization error (2.2) can be obtained easily by (3.1) and (3.2), which is expressed as follows:

$$
\begin{aligned}
\dot{e} & =\dot{x}-\dot{H}(t) y-H(t) \dot{y} \\
& =A x+f(x)+D(x) \theta(t)-\dot{H}(t) y-H(t)[B y+g(y)+u(x, y, t)] \\
& =A x+f(x)+D(x) \Phi+D(x) \Delta \Phi(t)-\dot{H}(t) y-H(t) B y-H(t) g(y)-H(t) u(x, y, t) .
\end{aligned}
$$

According to (3.4), we select the controller in the following form:

$u(x, y, t)=H^{-1}(t)[e+A x+f(x)+D(x) \widehat{\Phi}(t)+\beta(e, x, \widehat{M}, t)-\dot{H}(t) y-H(t) B y-H(t) g(y)]$

where $\beta(e, x, \widehat{M}, t)$ is a compensator to be designed to compensate the time-varying uncertainties. $\widehat{\Phi}(t), \widehat{M}$ are updated by the updating laws of unknown parameters $\Phi$ and unknown upper bound $M$, respectively. Then, (3.4) can be formulated as

$$
\dot{e}=-e+D(x) \tilde{\Phi}+D(x) \Delta \Phi(t)-\beta(e, x, \widehat{M}, t)
$$

where $\widetilde{\Phi}(t)=\Phi-\widehat{\Phi}(t)$ and $\widehat{M}$ is an estimation of unknown constant $M$ in (3.3).

Note the synchronization of two chaotic dynamical systems is essentially equivalent to stabilizing their corresponding error dynamical system at the origin; that is to say, two chaotic systems synchronize if the zero solution of their error system is asymptotically stable. In this paper, the robust adaptive controller should satisfy that the solution of (3.6) is stable at $e=0$.

We choose the Lyapunov function

$$
V(t)=\frac{1}{2} e^{\mathrm{T}}(t) e(t)+\frac{1}{2} \widetilde{\Phi}^{\mathrm{T}}(t) \Gamma_{1}^{-1} \widetilde{\Phi}(t)+\frac{1}{2 \Gamma_{2}} \widetilde{M}^{2}(t),
$$

where $\widetilde{M}=M-\widehat{M}, \Gamma_{1}^{\mathrm{T}}=\Gamma_{1}>0, \Gamma_{2}>0$ are adaptive gains. If $V(t)$ is positive definite and $\dot{V}(t)$ is negative definite, the synchronization will be achieved.

Theorem 3.6. Under Assumption 3.4, for a given synchronization scaling function matrix $H(t)$ and any initial conditions $x(0), y(0)$, there is a compensator

$$
\beta(e, x, \widehat{M}, t)=\widehat{M} \frac{D(x) D^{T}(x) e}{\left\|e^{T}(t) D(x)\right\|} \vartheta(t)
$$


and parameters updating law

$$
\begin{gathered}
\dot{\widehat{\Phi}}=\Gamma_{1} D^{\mathrm{T}}(x) e(t), \\
\dot{\bar{M}}=\Gamma_{2} \vartheta(t)\left\|e^{\mathrm{T}}(t) D(x)\right\|
\end{gathered}
$$

such that the error system (3.6) is globally stable. Then, the HFPS of different chaotic systems is achieved under the control law (3.5), the compensator (3.8), and the parameter updating law (3.9).

Proof. For (3.6) and (3.9), select the Lyapunov function as (3.7). Then its derivative along the error dynamical system (3.6) is

$$
\begin{aligned}
\dot{V}(t)= & e^{\mathrm{T}}(t) \dot{e}(t)+\tilde{\Phi}^{\mathrm{T}} \Gamma_{1}^{-1} \dot{\tilde{\Phi}}+\frac{1}{\Gamma_{2}} \widetilde{M} \dot{\bar{M}} \\
= & e^{\mathrm{T}}(t)(-e(t)+D(x) \tilde{\Phi}+D(x) \Delta \Phi(t)-\beta(e, x, \widehat{M}, t))-\tilde{\Phi}^{\mathrm{T}} \Gamma_{1}^{-1} \dot{\hat{\Phi}}-\frac{1}{\Gamma_{2}} \widetilde{M} \dot{\bar{M}} \\
= & -e^{\mathrm{T}}(t) e(t)+e^{\mathrm{T}}(t) D(x) \tilde{\Phi}+e^{\mathrm{T}}(t) D(x) \Delta \Phi(t)-e^{\mathrm{T}}(t) \beta(e, x, \widehat{M}, t) \\
& -\widetilde{\Phi}^{\mathrm{T}} D^{\mathrm{T}}(x) e(t)-\frac{1}{\Gamma_{2}} \widetilde{M} \dot{\bar{M}} \\
= & -e^{\mathrm{T}}(t) e(t)+e^{\mathrm{T}}(t) D(x) \Delta \Phi(t)-e^{\mathrm{T}}(t) \beta(e, x, \widehat{M}, t)-\frac{1}{\Gamma_{2}} \widetilde{M} \dot{\bar{M}} \\
\leq & -e^{\mathrm{T}}(t) e(t)+\left\|e^{\mathrm{T}}(t) D(x)\right\|\|\Delta \Phi(t)\|-e^{\mathrm{T}}(t) \beta(e, x, \widehat{M}, t)-\frac{1}{\Gamma_{2}} \widetilde{M} \dot{\bar{M}} \\
\leq & -e^{\mathrm{T}}(t) e(t)+\left\|e^{\mathrm{T}}(t) D(x)\right\| M \vartheta(t)-e^{\mathrm{T}}(t) \beta(e, x, \widehat{M}, t)-\frac{1}{\Gamma_{2}} \widetilde{M} \dot{\bar{M}} .
\end{aligned}
$$

We note that

$$
\begin{aligned}
\left\|e^{\mathrm{T}}(t) D(x)\right\| M \vartheta(t)-e^{\mathrm{T}}(t) \beta(e, x, \widehat{M}, t) & =\left\|e^{\mathrm{T}}(t) D(x)\right\| M \vartheta(t)-\widehat{M} e^{\mathrm{T}}(t) \frac{D(x) D^{\mathrm{T}}(x) e}{\left\|e^{\mathrm{T}}(t) D(x)\right\|} \vartheta(t) \\
& =\left\|e^{\mathrm{T}}(t) D(x)\right\| M \vartheta(t)-\widehat{M} \frac{\left\|e^{\mathrm{T}}(t) D(x)\right\|^{2}}{\left\|e^{\mathrm{T}}(t) D(x)\right\|} \vartheta(t) \\
& =\left\|e^{\mathrm{T}}(t) D(x)\right\| M \vartheta(t)-\widehat{M}\left\|e^{\mathrm{T}}(t) D(x)\right\| \vartheta(t) \\
& =\widetilde{M}\left\|e^{\mathrm{T}}(t) D(x)\right\| \vartheta(t) .
\end{aligned}
$$

Thus, substituting (3.11) into (3.10), and from (3.9), we have

$$
\dot{V}(t) \leq-e^{\mathrm{T}}(t) e(t)
$$


Then

$$
\dot{V}(t)<0
$$

when $\|e(t)\| \neq 0$. Since $V(t)$ is positive definite and $\dot{V}(t)$ is negative definite, based on the Lyapunov stability theory, the error system (3.6) is asymptotically stable; this completes the proof.

Remark 3.7. Note that the scaling function matrix $H(t)$ has no effect on $\dot{V}(t)$. Thus, one can change the scaling function matrix arbitrarily during control without worrying about the control robustness.

Remark 3.8. As switching phenomenon occurs in the control signal (3.5), the control approach may lead to less feasibility in engineering applications. Usually, there exist two approaches to eliminate the scattering, one is choosing the saturation-type smooth control signal [25], another one is the use of the leakage-like adaptive law to prevent the parameters drift [26]. Considering the issue, we will give a modification strategy to deal with the problem.

Theorem 3.9. If Assumption 3.4 on system (3.1) is satisfied, and for given synchronization scaling function matrix $H(t)$ and any initial conditions $x(0), y(0)$, then the control law (3.5) with the following smooth compensator:

$$
\beta(e, x, \widehat{M}, t)=\widehat{M}^{2} \frac{D(x) D^{\mathrm{T}}(x) e}{\left\|e^{\mathrm{T}}(t) D(x)\right\| \widehat{M}+\delta} \vartheta(t)
$$

and parameters updating law with $\sigma$-modification

$$
\begin{gathered}
\dot{\hat{\Phi}}=\Gamma_{1}\left(D^{\mathrm{T}}(x) e(t)-\sigma_{1} \widehat{\Phi}\right), \\
\dot{\widehat{M}}=\Gamma_{2}\left(\vartheta(t)\left\|e^{\mathrm{T}}(t) D(x)\right\|-\sigma_{2} \widehat{M}\right),
\end{gathered}
$$

where $\delta>0$ and $\sigma_{1}>0, \sigma_{2}>0$ guarantees that all the signals are bounded and the synchronization errors of (3.4) are UUB.

Proof. Choose the Lyapunov function as (3.7); again, similar to the derivation of (3.10), its derivative along the error dynamical system (3.6) and parameters updating law (3.15) is

$$
\dot{V}(t) \leq-e^{\mathrm{T}}(t) e(t)+\left\|e^{\mathrm{T}}(t) D(x)\right\| M \vartheta(t)-e^{\mathrm{T}}(t) \beta(e, x, \widehat{M}, t)+\sigma_{1} \tilde{\Phi}^{\mathrm{T}} \widehat{\Phi}-\frac{1}{\Gamma_{2}} \widetilde{M} \dot{\bar{M}}
$$


From (3.14), we have the following inequality:

$$
\begin{aligned}
& \left\|e^{\mathrm{T}}(t) D(x)\right\| M \vartheta(t)-e^{\mathrm{T}}(t) \beta(e, x, \widehat{M}, t) \\
& =\left\|e^{\mathrm{T}}(t) D(x)\right\| M \vartheta(t)-\widehat{M}^{2} \frac{e^{\mathrm{T}} D(x) D^{\mathrm{T}}(x) e}{\left\|e^{\mathrm{T}}(t) D(x)\right\| \widehat{M}+\delta} \vartheta(t) \\
& =\left\|e^{\mathrm{T}}(t) D(x) \widetilde{M} \vartheta(t)+\right\| e^{\mathrm{T}}(t) D(x) \| \widehat{M} \vartheta(t)-\widehat{M}^{2} \frac{e^{\mathrm{T}} D(x) D^{\mathrm{T}}(x) e}{\left\|e^{\mathrm{T}}(t) D(x)\right\| \widehat{M}+\delta} \vartheta(t) \\
& =\left\|e^{\mathrm{T}}(t) D(x)\right\| \widetilde{M} \vartheta(t)+\frac{\delta\left\|e^{\mathrm{T}} D(x)\right\| \widehat{M} \vartheta(t)}{\left\|e^{\mathrm{T}}(t) D(x)\right\| \widehat{M}+\delta} \\
& \leq\left\|e^{\mathrm{T}}(t) D(x)\right\| \widetilde{M} \vartheta(t)+\frac{\delta\left\|e^{\mathrm{T}} D(x)\right\| \widehat{M} \vartheta(t)}{\left\|e^{\mathrm{T}}(t) D(x)\right\| \widehat{M}} \\
& \leq\left\|e^{\mathrm{T}}(t) D(x)\right\| \widetilde{M} \vartheta(t)+\delta \vartheta(t) .
\end{aligned}
$$

Substituting (3.17) into (3.16), we get

$$
\dot{V}(t) \leq-e^{\mathrm{T}}(t) e(t)-\sigma_{1} \tilde{\Phi}^{\mathrm{T}} \widetilde{\Phi}+\sigma_{1} \widetilde{\Phi}^{\mathrm{T}} \Phi-\sigma_{2} \widetilde{M}^{2}+\sigma_{2} \widetilde{M} M+\delta \vartheta(t)
$$

Using $2 a b \leq a^{2}+b^{2}$ for any positive constants $a, b$, we can obtain

$$
\dot{V}(t) \leq-e^{\mathrm{T}}(t) e(t)-\frac{\sigma_{1}}{2 \widetilde{\Phi}^{\mathrm{T}} \widetilde{\Phi}}-\frac{\sigma_{2}}{2 \widetilde{M}^{2}}+\frac{\sigma_{1}}{2 \Phi^{\mathrm{T}} \Phi}+\frac{\sigma_{2}}{2 M^{2}}+\bar{\delta}
$$

where $\bar{\delta}$ is an upper bound of $\delta \vartheta(t)$ for all $t \geq 0$.

From (3.7) and (3.19), we have

$$
\dot{V}(t) \leq-c V(t)+\bar{\epsilon}
$$

where

$$
c=\min \left(2, \sigma_{1} \lambda_{\max }\left(\Gamma_{1}\right), \sigma_{2} \Gamma_{2}\right), \quad \bar{\epsilon}=\bar{\delta}+\frac{\sigma_{1}\|\Phi\|^{2}}{2}+\frac{\sigma_{2} M^{2}}{2}
$$

with $\lambda_{\max }\left(\Gamma_{1}\right)$ denot the maximum eigenvalue of $\Gamma_{1}$. It is obvious that $\dot{V}(t) \leq 0$, whenever $V(t) \geq \bar{\epsilon} / c$. Thus, $V(t) \leq k$, with $k>\bar{\epsilon} / c>0$, is an invariant set; that is, if $V\left(t_{0}\right) \leq k$, then $V(t) \leq k$ for all $t \geq t_{0}$.

By the comparison principle, from (3.20), we can get

$$
0 \leq V(t) \leq \frac{\bar{\epsilon}}{c}+\left(V\left(t_{0}\right)-\frac{\bar{\epsilon}}{c}\right) e^{-c t}
$$


From the definition of $V(t)$ in (3.7), the synchronization errors are bounded by

$$
\|e(t)\| \leq \sqrt{\max \left(V\left(t_{0}\right), \frac{\bar{\epsilon}}{c}\right)} .
$$

Equation (3.22)means that $V(t)$ is ultimately bounded by $\bar{\epsilon} / c$. Thus, signals $e(t), \widetilde{\Phi}, \widetilde{M}$ are uniformly ultimately bounded (UUB).

Remark 3.10. It is easy to see that the design parameters $\delta$ and $\sigma_{1}, \sigma_{2}$ determine the final accuracy of the synchronization errors, which can be arbitrarily small provided the design parameter $\delta$ and upper bound of system parameters $M, \Phi$ are small enough. As expected, the smaller the desired errors, the larger the controllers' gain.

Corollary 3.11. If Assumption 3.4 on the time-varying parameters of system (3.1) is changed as follows

$$
\|\theta(t)\| \leq M_{0} \vartheta(t)
$$

for all $t \in R^{+}$, where $M_{0} \in R^{+}$is the unknown constant parameter, $\vartheta(t)$ is a known continuous bounded function of $t$. For given synchronization scaling function matrix $H(t)$ and any initial conditions $x(0), y(0)$, the control law

$$
u(x, y, t)=H^{-1}(t)\left[e+A x+f(x)+\beta\left(e, x, \widehat{M}_{0}, t\right)-\dot{H}(t) y-H(t) B y-H(t) g(y)\right]
$$

with the following compensator:

$$
\beta\left(e, x, \widehat{M}_{0}, t\right)=\widehat{M}_{0} \frac{D(x) D^{\mathrm{T}}(x) e}{\left\|e^{\mathrm{T}}(t) D(x)\right\|} \vartheta(t)
$$

and parameters updating law

$$
\dot{\widehat{M}}_{0}=\Gamma_{2} \vartheta(t)\left\|e^{\mathrm{T}}(t) D(x)\right\|, \quad \Gamma_{2}>0,
$$

can guarantee that the error system (3.6) is globally stable. Then, the HFPS of different chaotic systems is achieved under the control law (3.25), the compensator (3.26), and the parameter updating law (3.27).

Corollary 3.12. If Assumption 3.4 on the time-varying parameters of system (3.1) is changed as (3.24), the controller law (3.25) with the following smooth compensator:

$$
\beta\left(e, x, \widehat{M}_{0}, t\right)=\widehat{M}_{0}^{2} \frac{D(x) D^{\mathrm{T}}(x) e}{\left\|e^{\mathrm{T}}(t) D(x)\right\| \widehat{M}_{0}+\delta} \vartheta(t)
$$


and parameters updating law

$$
\dot{\bar{M}}_{0}=\Gamma_{2}\left(\vartheta(t)\left\|e^{T}(t) D(x)\right\|-\sigma_{2} \widehat{M}_{0}\right), \quad \sigma_{2}>0, \Gamma_{2}>0,
$$

can guarantee that all the signals are bounded and the synchronization errors of (3.4) are UUB, and upper bound of UUB can be arbitrarily small provided the design parameter $\delta$ and upper bound of time-varying parameters $M_{0}$ are small enough.

\section{Simulation Results}

In this section, two examples are presented to show the effectiveness of the proposed robust adaptive controllers.

Example 4.1. Consider the hybrid function projective synchronization between Lorenz system

$$
\begin{gathered}
\dot{x}_{1}=\theta_{1}(t)\left(x_{2}-x_{1}\right), \\
\dot{x}_{2}=-x_{1} x_{3}-x_{2}+\theta_{2}(t) x_{1}, \\
\dot{x}_{3}=x_{1} x_{2}-\theta_{3}(t) x_{3}
\end{gathered}
$$

and the Chen system

$$
\begin{gathered}
\dot{y}_{1}=35\left(y_{2}-y_{1}\right)+u_{1}, \\
\dot{y}_{2}=-y_{1} y_{3}+28 y_{2}-7 y_{1}+u_{2}, \\
\dot{y}_{3}=y_{1} y_{2}-3 y_{3}+u_{3} .
\end{gathered}
$$

Comparing system (4.1) and (4.2) with (3.1) and (3.2), we obtain

$$
\begin{gathered}
A=\left[\begin{array}{ccc}
0 & 0 & 0 \\
0 & -1 & 0 \\
0 & 0 & 0
\end{array}\right], \quad f(x)=\left(\begin{array}{c}
0 \\
-x_{1} x_{3} \\
x_{1} x_{2}
\end{array}\right), \quad D(x)=\left[\begin{array}{ccc}
x_{2}-x_{1} & 0 & 0 \\
0 & x_{1} & 0 \\
0 & 0 & -x_{3}
\end{array}\right], \\
B=\left[\begin{array}{ccc}
-35 & 35 & 0 \\
-7 & 28 & 0 \\
0 & 0 & -3
\end{array}\right], \quad g(y)=\left(\begin{array}{c}
0 \\
-y_{1} y_{3} \\
y_{1} y_{2}
\end{array}\right)
\end{gathered}
$$

and the unknown uncertain parameter vector $\theta(t)=\left[10+\rho_{1} \sin t 28+\rho_{2} \cos t 8 / 3-\rho_{3} \sin t\right]^{\mathrm{T}}$. If the system parameters are chosen to be $\theta_{1}(t)=10, \theta_{2}(t)=28, \theta_{3}(t)=8 / 3$, then the Lorenz system has a chaotic attractor. We obtain the nominal value of the parameter vector $\Phi=\left[\begin{array}{lll}10 & 28 & 8 / 3\end{array}\right]^{\mathrm{T}}$. And hence $\Delta \Phi=\theta(t)-\Phi=\left[\rho_{1} \sin t \rho_{2} \cos t \rho_{3} \sin t\right]^{\mathrm{T}}$. We suppose that 

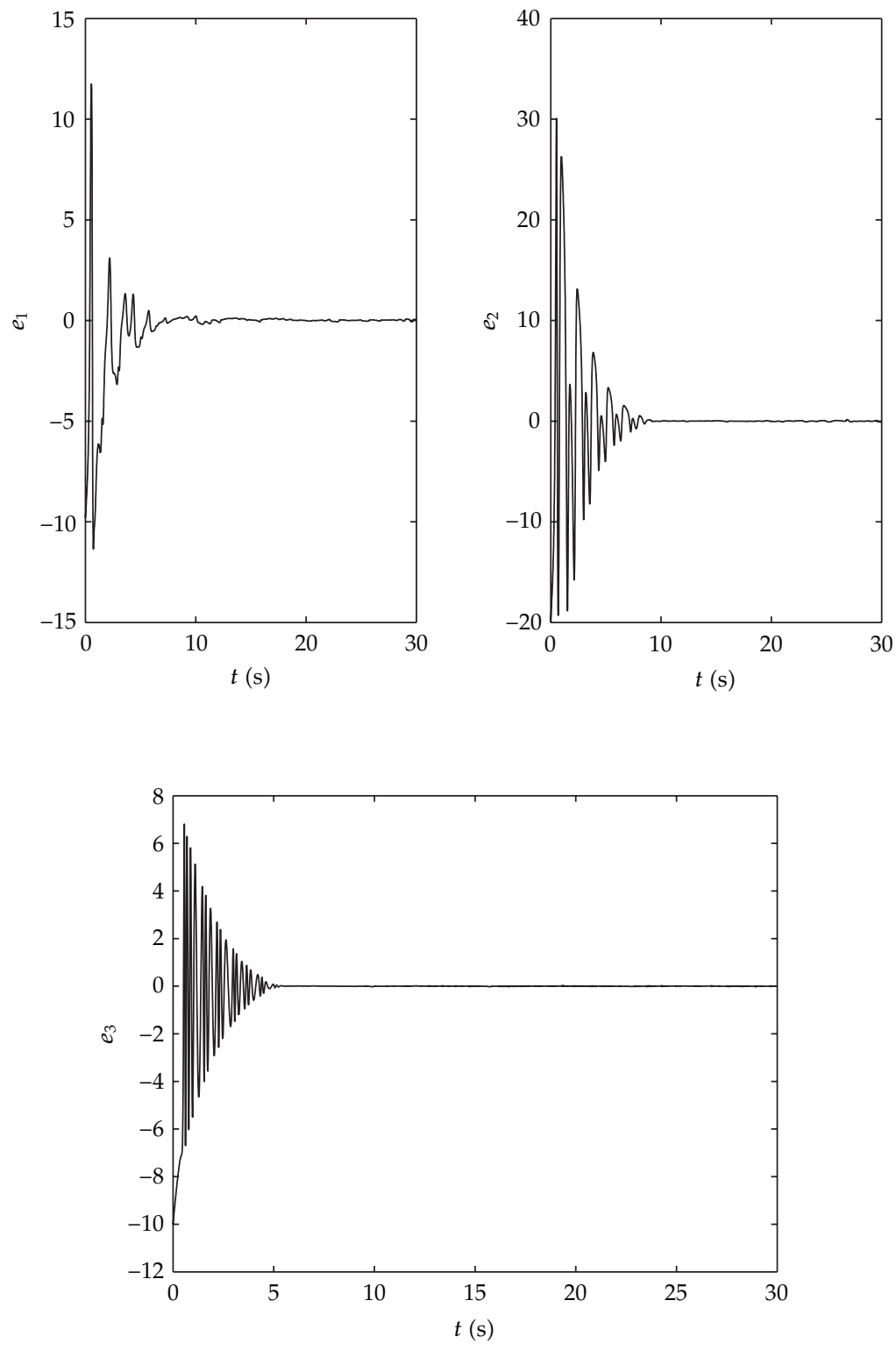

Figure 1: The time evolution of the HFPS errors.

the upper bound of norm of $\Delta \Phi$ can be derived as $M \vartheta(t)$, where $M$ is assumed to be an unknown parameter and $\vartheta(t)=\sqrt{1+(\sin t)^{2}}$, and the initial estimated values of the unknown parameters $\Phi$ are $\widehat{\Phi}(0)=\left[\begin{array}{lll}0 & 0 & 0\end{array}\right]^{\mathrm{T}}$, and $\widehat{M}(0)=0$. The initial states of the drive system and

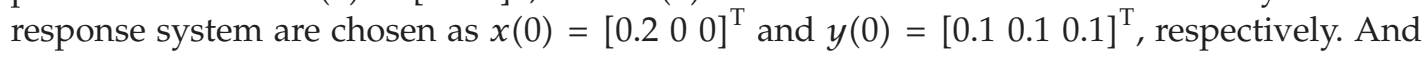
choose adaptive gains as $\Gamma_{1}=0.01 I_{3}, \Gamma_{2}=1, \delta=0.06, \sigma_{1}=0.1, \sigma_{2}=0.05$. By taking the 

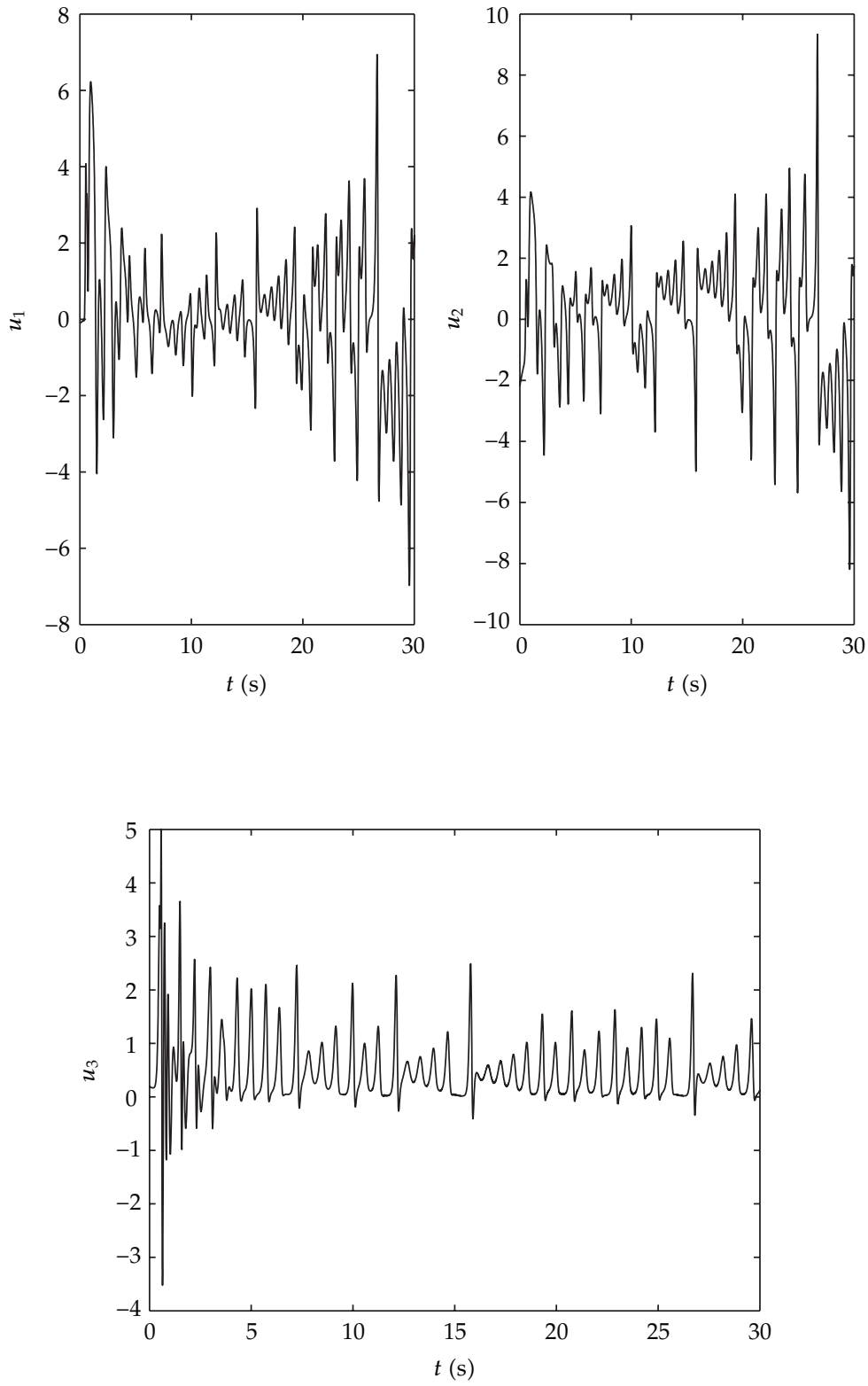

Figure 2: The time evolution of the controllers.

scaling function matrix as $H(t)=\operatorname{diag}\{100+100 \sin (2 \pi t / 99), 100+100 \cos (2 \pi t / 99), 100+$ $100 \sin (2 \pi / 99)\}$ according to the Theorem 3.9, we conclude that the controller $u$ along with the parameter updating law given by (3.5), (3.14), and (3.15) will achieve the hybrid function projective synchronization of the Lorenz system and Chen system systems. This is verified by the simulation results shown in Figure 1. Furthermore, Figure 2 depicts the time evolution of the controllers, and Figure 3 shows the evolution of the estimated parameters. 

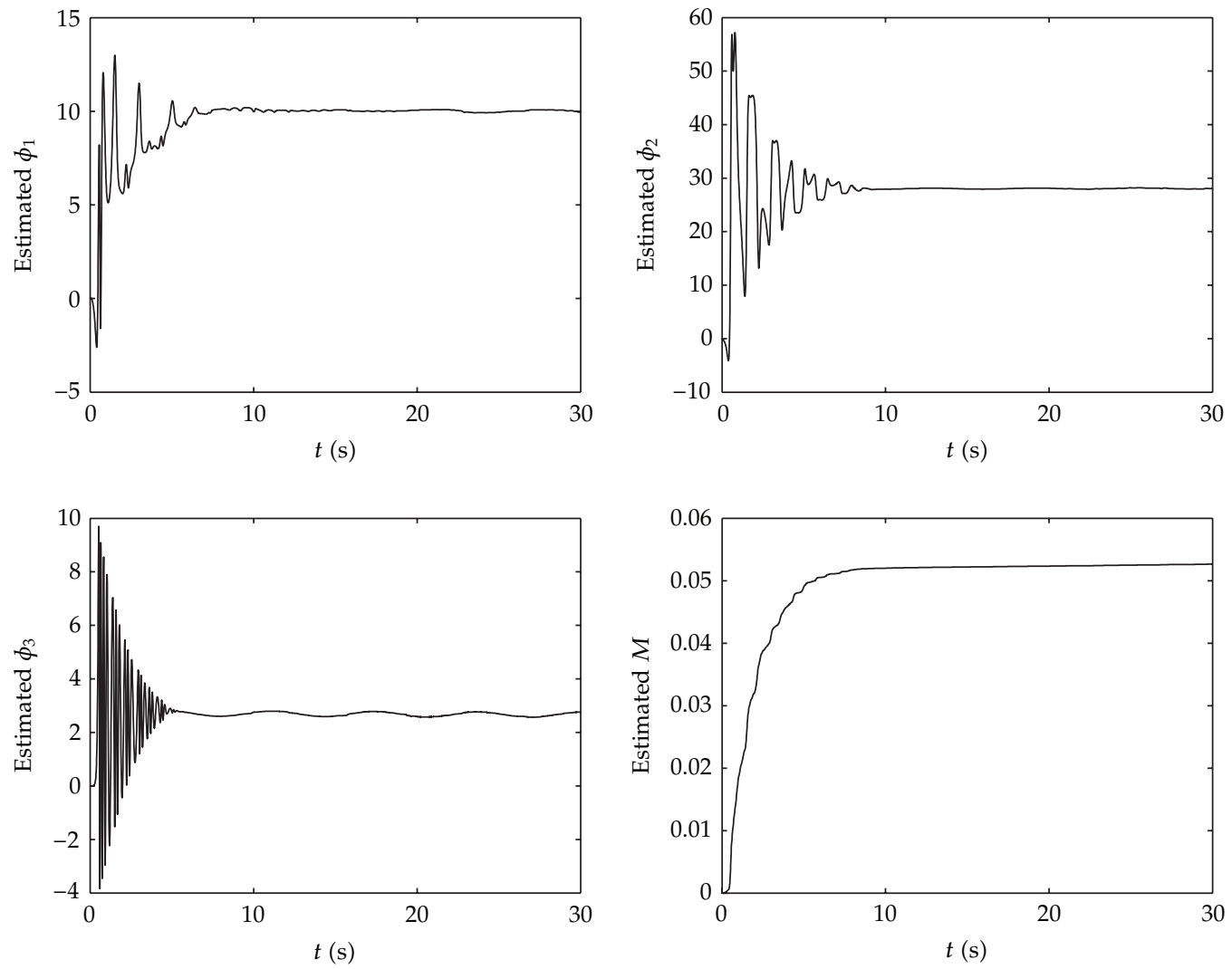

Figure 3: The time evolution of the estimated parameters.

Example 4.2. Consider the hybrid function projective synchronization between hyperchaotic Chen systems

$$
\begin{gathered}
\dot{x}_{1}=\theta_{1}(t)\left(x_{2}-x_{1}\right)+x_{4}, \\
\dot{x}_{2}=-x_{1} x_{3}+\theta_{2}(t) x_{1}+\theta_{3}(t) x_{2}, \\
\dot{x}_{3}=x_{1} x_{2}-\theta_{4}(t) x_{3}, \\
\dot{x}_{4}=x_{2} x_{3}-\theta_{5}(t) x_{4}
\end{gathered}
$$

and the generalized Lorenz system

$$
\begin{gathered}
\dot{y}_{1}=y_{2}-y_{1}+1.5 y_{4}+u_{1}, \\
\dot{y}_{2}=-y_{1} y_{3}+26 y_{1}-y_{2}+u_{2}, \\
\dot{y}_{3}=y_{1} y_{2}-0.7 y_{3}+u_{3}, \\
\dot{y}_{4}=-y_{1}-y_{4}+u_{4} .
\end{gathered}
$$



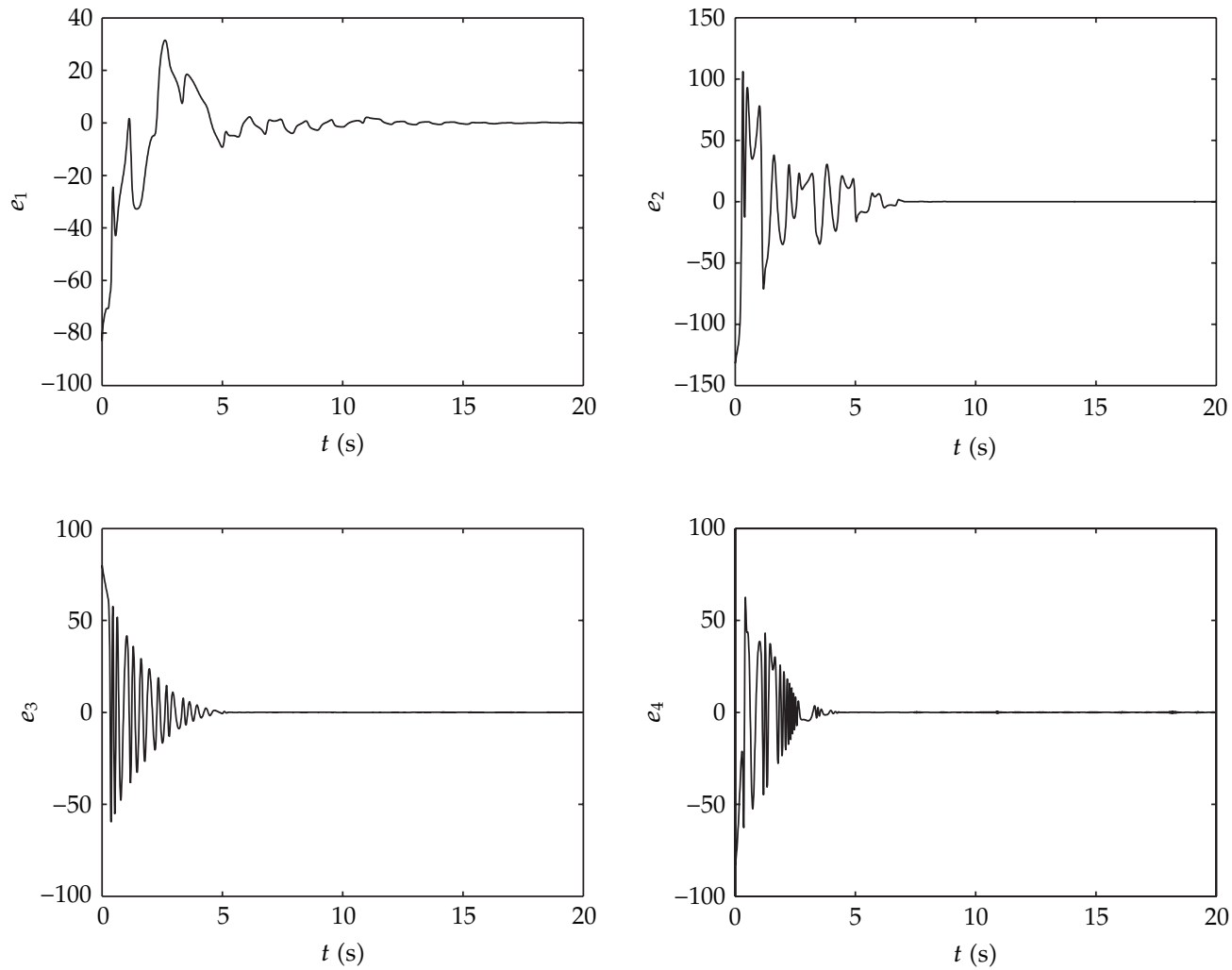

Figure 4: The time evolution of the HFPS errors.

Comparing system (4.4) and (4.5) with (3.1) and (3.2), we get that

$$
\begin{gathered}
A=\left[\begin{array}{llll}
0 & 0 & 0 & 1 \\
0 & 0 & 0 & 0 \\
0 & 0 & 0 & 0 \\
0 & 0 & 0 & 0
\end{array}\right], \quad f(x)=\left(\begin{array}{c}
0 \\
-x_{1} x_{3} \\
x_{1} x_{2} \\
x_{2} x_{3}
\end{array}\right), \\
D(x)=\left[\begin{array}{ccccc}
x_{2}-x_{1} & 0 & 0 & 0 & 0 \\
0 & x_{1} & x_{2} & 0 & 0 \\
0 & 0 & 0 & -x_{3} & 0 \\
0 & 0 & 0 & 0 & -x_{4}
\end{array}\right], \\
B=\left[\begin{array}{cccc}
-1 & 1 & 0 & 1.5 \\
26 & -1 & 0 & 0 \\
0 & 0 & -0.7 & 0 \\
-1 & 0 & 0 & -1
\end{array}\right], \quad g(y)=\left(\begin{array}{c}
0 \\
-y_{1} y_{3} \\
y_{1} y_{2} \\
0
\end{array}\right) .
\end{gathered}
$$



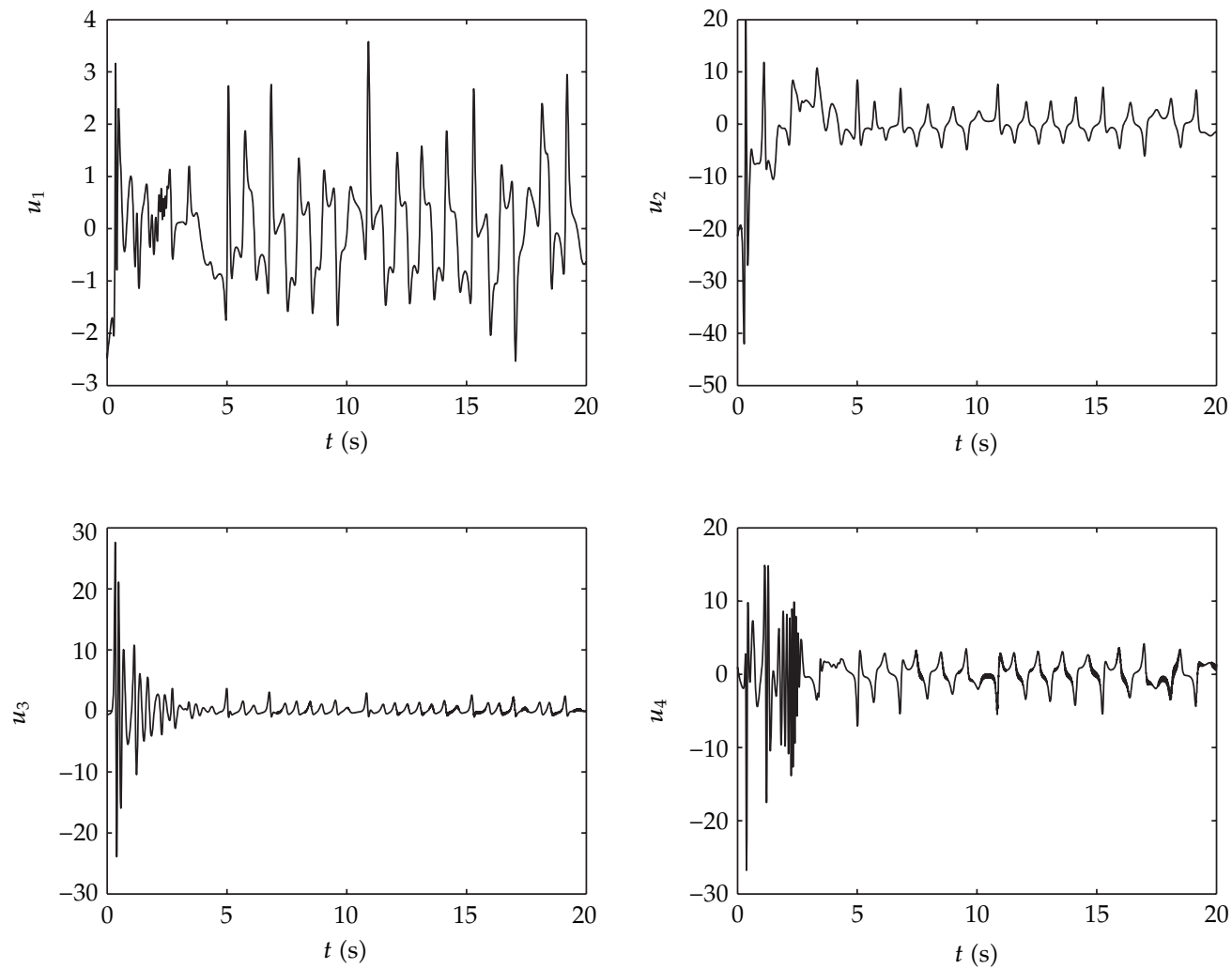

Figure 5: The time evolution of the controllers.

The unknown time-varying parameter vector is $\theta(t)=\left[35+\rho_{1} \sin t 7+\rho_{2} \cos t 12-\rho_{3} \sin t 3+\right.$ $\left.\rho_{4} \cos t 0.5-\rho_{5} \sin t\right]^{\mathrm{T}}$. If the system parameters are chosen to be $\theta_{1}(t)=35, \theta_{2}(t)=7$, $\theta_{3}(t)=12, \theta_{4}(t)=3, \theta_{5}(t)=0.5$, then hyper-chaotic Chen systems have chaotic attractor. We get the nominal value of the parameter vector as $\Phi=\left[\begin{array}{lllll}35 & 7 & 12 & 3 & 0.5\end{array}\right]^{\mathrm{T}}$. Thus, $\Delta \Phi=$ $\theta(t)-\Phi=\left[\begin{array}{lll}\rho_{1} \sin t & \rho_{2} \cos t-\rho_{3} \sin t \quad \rho_{4} \cos t-\rho_{5} \sin t\end{array}\right]^{\mathrm{T}}$. We suppose that the upper bound of norm of $\Delta \Phi$ can be derived as $M \vartheta(t)$, where $M$ is assumed to be an unknown parameter and $\vartheta(t)=\sqrt{2+(\sin t)^{2}}$. The initial estimated values of the unknown parameters $\Phi$ are $\widehat{\Phi}(0)=\left[\begin{array}{llll}0 & 0 & 0 & 0\end{array}\right]^{\mathrm{T}}$ and $\widehat{M}(0)=0$. The initial states of the drive system and response system are chosen as $x(0)=\left[\begin{array}{llll}-3 & 0 & 0 & 5\end{array}\right]^{\mathrm{T}}$ and $y(0)=\left[\begin{array}{lllll}0.8 & 1.2 & -0.8 & 0.8\end{array}\right]^{\mathrm{T}}$, respectively. And choose adaptive gains as $\Gamma_{1}=0.002 I_{5}, \Gamma_{2}=1$. By taking the scaling function matrix as $H(t)=100 \operatorname{diag}\{1+\sin (2 \pi t / 120), 1+\cos (2 \pi t / 120), 1-\sin (\pi t / 120), 1-\cos (2 \pi t / 120), 1-$ $\cos (\pi t / 120)\}$, according to Theorem 3.6, we conclude that the control vector $u$ along with the parameter updating law given by (3.5), (3.8), and (3.9) will achieve the adaptive hybrid function projective synchronization of the hyperchaotic Chen system and the generalized Lorenz system, as verified by the simulation results shown in Figure 4. Furthermore, Figure 5 depicts the time evolution of the controllers, and Figure 6 shows the evolution of the estimated parameters. 

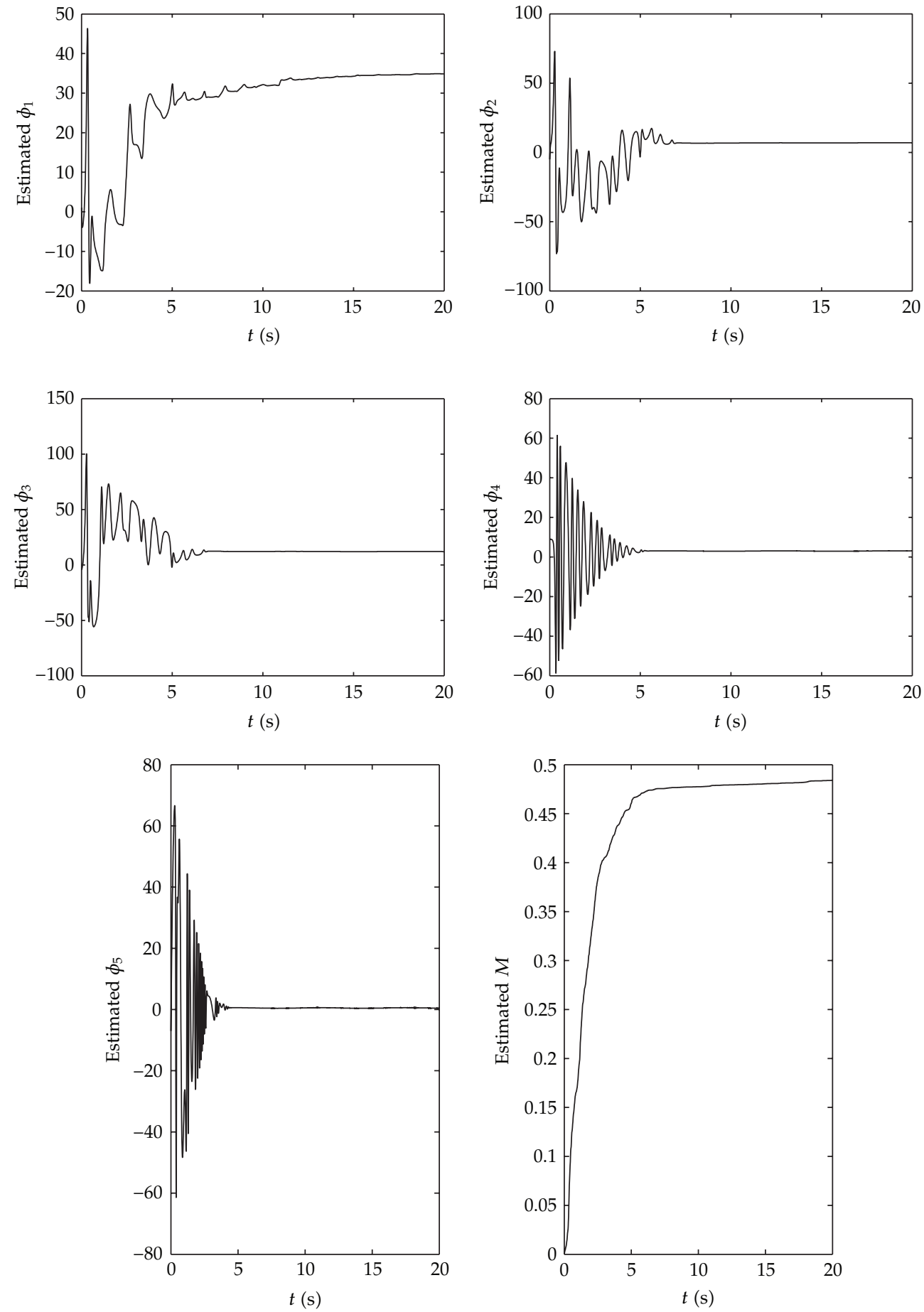

Figure 6: The time evolution of the estimated parameters. 


\section{Conclusions}

In this paper, we have introduced the definition of AHFPS and given the AHFPS scheme of a class chaotic system with unknown time varying parameters. Based on the Lyapunov stability theory, a robust adaptive controller and the parameter update law are obtained for the stability of the error dynamics between the drive and the response systems. This controller can be applied to more critical conditions, where the parameters are unknown time-varying and where there are also uncertainties in the parameters. We need not know the parameters thoroughly if the uncertainties of the parameters are bounded by the product of a known function of $t$ and an unknown constant. The proposed controllers have been applied to the Chen system and the Lorenz system, the hyper-chaotic Chen system, and the generalized Lorenz system. The simulation results show the effective performance of the proposed synchronization.

\section{Acknowledgment}

This work was supported by the Soft Science Foundation of Shanxi province (2011041033-3).

\section{References}

[1] L. M. Pecora and T. L. Carroll, "Synchronization in chaotic systems," Physical Review Letters, vol. 64, no. 8, pp. 821-824, 1990.

[2] C. D. Li, X. F. Liao, and R. Zhang, "Impulsive synchronization of nonlinear coupled chaotic systems," Physics Letters A, vol. 328, no. 1, pp. 47-50, 2004.

[3] A. E. Hramov and A. A. Koronovskii, "Generalized synchronization: a modified system approach," Physical Review E, vol. 71, no. 6, Article ID 067201, 2005.

[4] E. M. Shahverdiev, S. Sivaprakasam, and K. A. Shore, "Lag synchronization in time-delayed systems," Physics Letters A, vol. 292, no. 6, pp. 320-324, 2002.

[5] C. Masoller, "Anticipation in the synchronization of chaotic time-delay systems," Physica A, vol. 295, no. 1-2, pp. 301-304, 2001.

[6] S. Banerjee, P. Saha, and A. R. Chowdhury, "On the application of adaptive control and phase synchronization in non-linear fluid dynamics," International Journal of Non-Linear Mechanics, vol. 39, no. 1, pp. 25-31, 2004.

[7] W. Q. Liu, “Antiphase synchronization in coupled chaotic oscillators," Physical Review E, vol. 73, no. 5, Article ID 057203, 2006.

[8] J. H. Park, "Chaos synchronization between two different chaotic dynamical systems," Chaos, Solitons and Fractals, vol. 27, no. 2, pp. 549-554, 2006.

[9] X. Huang, J. Cao, and D. W. Ho, "Synchronization criteria for lur'e systems by dynamic output feedback with time-delay," International Journal of Bifurcation and Chaos, vol. 16, no. 8, pp. 2293-2307, 2006.

[10] M. T. Yassen, "Adaptive chaos control and synchronization for uncertain new chaotic dynamical system," Physics Letters A, vol. 350, no. 1-2, pp. 36-43, 2006.

[11] R.-Z. Luo, "Impulsive control and synchronization of a new chaotic system," Physics Letters A, vol. 372, no. 5, pp. 648-653, 2008.

[12] J. A. Lu, X. G. Wu, and J. Lü, "Synchronization of a unified chaotic system and the application in secure communication," Physics Letters A, vol. 305, no. 6, pp. 365-370, 2002.

[13] D. Xu and Z. Li, "Controlled projective synchronization in nonpartially-linear chaotic systems," International Journal of Bifurcation and Chaos in Applied Sciences and Engineering, vol. 12, no. 6, pp. 1395 1402, 2002.

[14] H. Y. Du, Q. S. Zeng, and C. G. Wang, "Function projective synchronization of different chaotic systems with uncertain parameters," Physics Letters A, vol. 372, no. 33, pp. 5402-5410, 2008.

[15] E. M. Elabbasy, H. N. Agiza, and M. M. El-Dessoky, "Adaptive synchronization of a hyperchaotic system with uncertain parameter," Chaos, Solitons and Fractals, vol. 30, no. 5, pp. 1133-1142, 2006. 
[16] R. H. Li, W. Xu, and S. Li, "Adaptive generalized projective synchronization in different chaotic systems based on parameter identification," Physics Letters A, vol. 367, no. 3, pp. 199-206, 2007.

[17] Y. W. Wang, C. Y. Wen, M. Yang, and J. W. Xiao, "Adaptive control and synchronization for chaotic systems with parametric uncertainties," Physics Letters A, vol. 372, no. 14, pp. 2409-2414, 2008.

[18] J. H. Park, "Adaptive modified projective synchronization of a unified chaotic system with an uncertain parameter," Chaos, Solitons and Fractals, vol. 34, no. 5, pp. 1552-1559, 2007.

[19] H. Salarieh and M. Shahrokhi, "Adaptive synchronization of two different chaotic systems with time varying unknown parameters," Chaos, Solitons and Fractals, vol. 37, no. 1, pp. 125-136, 2008.

[20] R. H. Li, "A special full-state hybrid projective synchronization in symmetrical chaotic systems," Applied Mathematics and Computation, vol. 200, no. 1, pp. 321-329, 2008.

[21] M. F. Hu, Y. Q. Yang, Z. Y. Xu, and L. X. Guo, "Hybrid projective synchronization in a chaotic complex nonlinear system," Mathematics and Computers in Simulation, vol. 79, no. 3, pp. 449-457, 2008.

[22] M. F. Hu, Z. Y. Xu, R. Zhang, and A. H. Hu, “Adaptive full state hybrid projective synchronization of chaotic systems with the same and different order," Physics Letters A, vol. 365, no. 4, pp. 315-327, 2007.

[23] K. Sudheer and M. Sabir, "Adaptive modified function projective synchronization between hyperchaotic Lorenz system and hyperchaotic Lu system with uncertain parameters," Physics Letters A, vol. 373, no. 41, pp. 3743-3748, 2009.

[24] Y. P. Sun, J.-M. Li, J.-A. Wang et al., "Generalized projective synchronization of chaotic systems via adaptive learning control," Chinese Physics B, vol. 19, no. 2, Article ID 020505, 2010.

[25] L. Q. Shen and M. Wang, "Robust synchronization and parameter identification on a class of uncertain chaotic systems," Chaos, Solitons and Fractals, vol. 38, no. 1, pp. 106-111, 2008.

[26] M. M. Arefi and M. R. Jahed-Motlagh, "Adaptive robust synchronization of Rossler systems in the presence of unknown matched time-varying parameters," Communications in Nonlinear Science and Numerical Simulation, vol. 15, no. 12, pp. 4149-4157, 2010. 


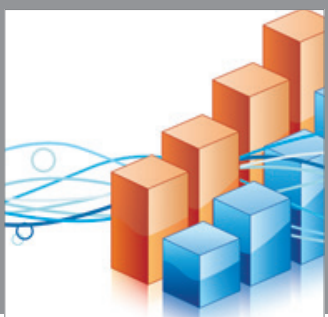

Advances in

Operations Research

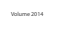

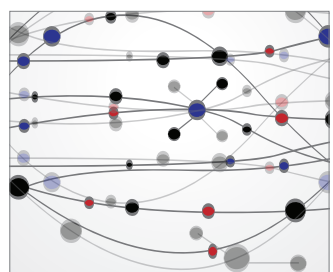

\section{The Scientific} World Journal
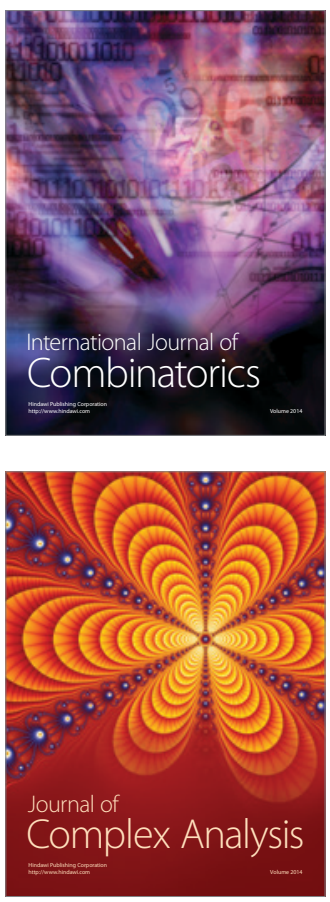

International Journal of

Mathematics and

Mathematical

Sciences
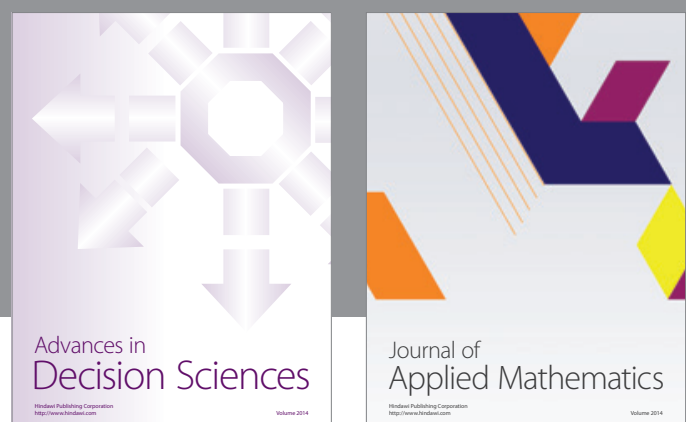

Journal of

Applied Mathematics
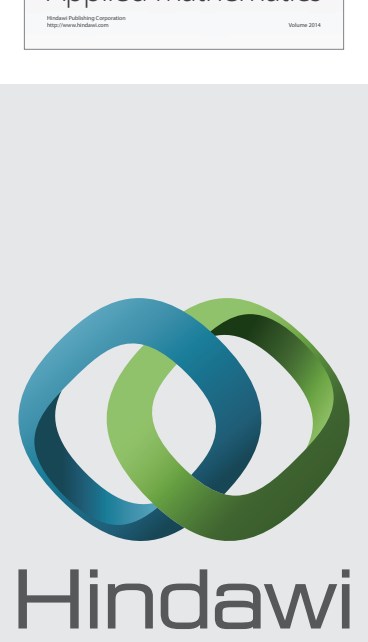

Submit your manuscripts at http://www.hindawi.com
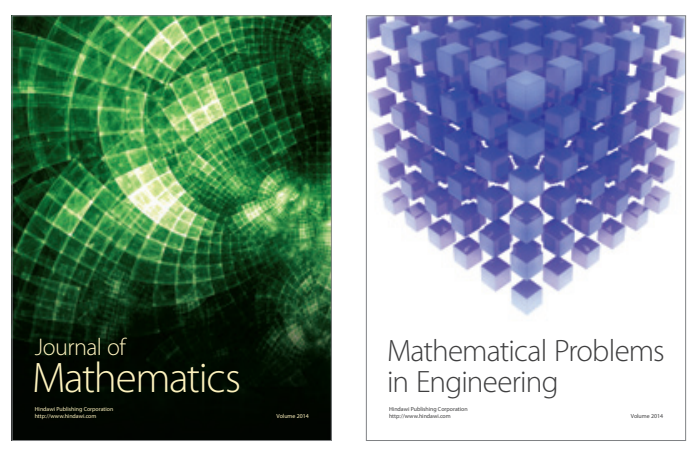

Mathematical Problems in Engineering
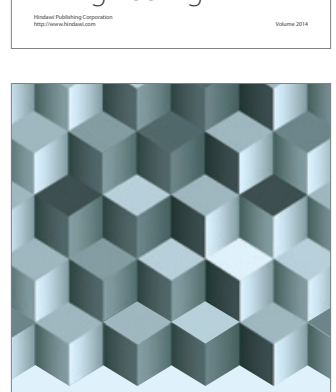

Journal of

Function Spaces
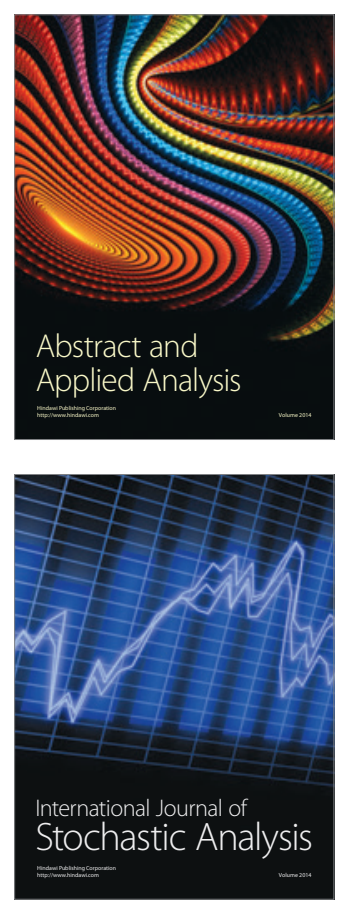

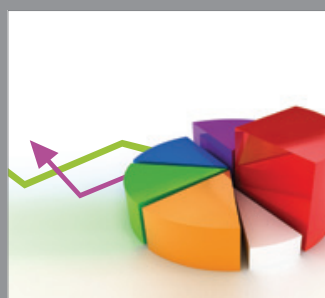

ournal of

Probability and Statistics

Promensencen
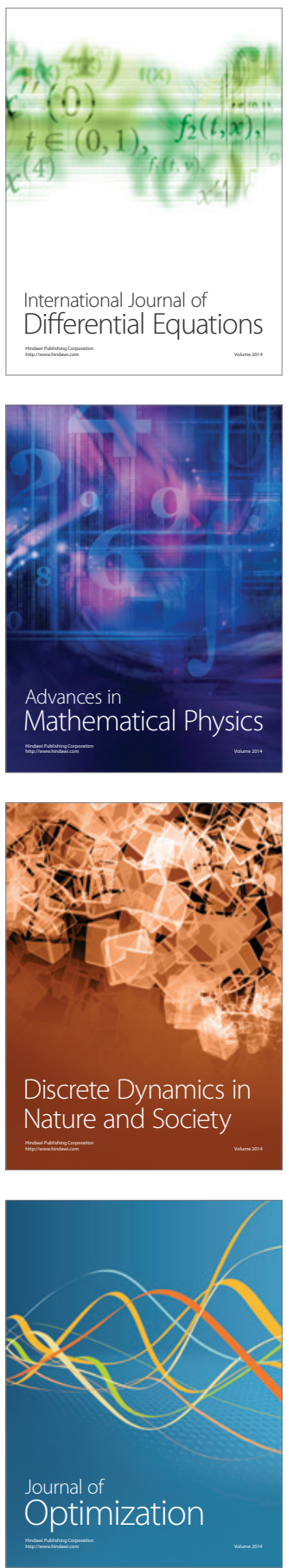